\title{
On infinite-dimensional dissipative quadratic stochastic operators
}

Farruh Shahidi*

\section{"Correspondence:}

farruh.shahidi@gmail.com

Department of Computer Science,

Faculty of Information and

Communication Technology,

International Islamic University

Malaysia, P.O. BOX 10, Kuala Lumpur,

50728, Malaysia

\begin{abstract}
The purpose of the paper is to extend the notion of dissipativity of maps on infinite-dimensional simplex. We study the fixed points of dissipative quadratic stochastic operators on infinite-dimensional simplex. Besides, we study the limit behavior of the trajectories of such operators. We also show the difference of dissipative operators defined on finite and infinite-dimensional spaces. We obtain the results by using majorization for infinite vectors and $\ell_{1}$ convergence.
\end{abstract}

MSC: 15A51;47H60;46T05; 92B99

Keywords: dissipative quadratic stochastic operators; infinite-dimensional space; majorization; trajectory; $\ell_{1}$-convergence

\section{Introduction}

A quadratic stochastic operators (q.s.o. in short), firstly initiated by Bernstein [1], is a nonlinear difference equation, which has arisen from some problems of population genetics. Further development of this theory belongs to Lyubich [2, 3], Kesten [4, 5], Vallander [6], and Zakharevich [7], where the authors investigate the limit behavior of the trajectories (or dynamics) of q.s.o. It should be noted that the limit behavior of the trajectories of q.s.o. on 1D simplex was fully studied by Lyubich $[2,3]$, where it was shown that the $\omega$-limit set (see definition below) of any initial point is a finite set. Vallander [6] studied the dynamics of some special q.s.o. on 2D simplex. Vallander's result was later extended to any finitedimensional space by Ganikhodzhaev in $[8,9]$. Later on, this special q.s.o. was called as Volterra q.s.o., which is, in fact the Lotka-Volterra predator prey equation in discrete settings. The dynamics of Volterra q.s.o. was somehow studied successfully in [8]. However, not all q.s.o. are of Volterra-type, and the dynamics of non-Volterra q.s.o. remains open. Notable results for non-Volterra q.s.o. were obtained by Rozikov and his students [10-13], who introduced different classes of q.s.o., such as 'strictly non-Volterra,' 'F-q.s.o.' (F stands for a 'female' due to its genetic interpretation), 'separable q.s.o.', ' $\ell$-Volterra' and studied the limit behavior of the trajectories. A manuscript [14] provides some results and open problem on q.s.o.

A majorization of vectors [15] turned out to be a useful tool for classifying q.s.o. into some of its subclasses. With the help of it, the definition of doubly stochastic and dissipative q.s.o. were introduced in papers [16] and [17], respectively. Further properties of such operators were studied in [18-20]. Of course, Volterra q.s.o. and classes considered in papers [10-13] are different from doubly stochastic and dissipative q.s.o. It is to note

( 2013 Shahidi; licensee Springer. This is an Open Access article distributed under the terms of the Creative Commons Attribution License (http://creativecommons.org/licenses/by/2.0), which permits unrestricted use, distribution, and reproduction in any medium, provided the original work is properly cited. 
that the limit behavior of the trajectories of the dissipative q.s.o. on finite-dimensional simplex (the set of vectors with non-negative components summing up to 1) was fully classified in [20]. Note that a q.s.o. is just a discrete probability distribution of a finite population. However, there are models where the probability distribution is countably infinite, which means that a q.s.o. is defined on infinite-dimensional space. In the simplest case, the infinite-dimensional space should be the Banach space $\ell_{1}$ of absolutely summable sequences. It is worth mentioning that Volterra q.s.o. and doubly stochastic q.s.o. on infinite-dimensional space was introduced and studied in papers [21] and [22], respectively.

Therefore, the purpose of the present paper is to introduce a dissipative q.s.o. on infinitedimensional subspace of $\ell_{1}$, by using majorization for infinite vectors [23]. We show the difference between finite- and infinite-dimensional cases. While the existence of fixed point and convergence of Cesaro averages (that is an ergodic theorem) holds for finitedimensional dissipative operators, we show that it fails for infinite-dimensional operators. We also provide some regular dissipative q.s.o. in infinite-dimensional case.

The paper is organized as follows. The next chapter provides some preliminaries and results from finite-dimensional cases. In Section 3, we introduce a dissipative q.s.o. in infinite-dimensional simplex and study its properties. Finally, we study the limit behavior of the trajectories of dissipative q.s.o. in Section 4. We use notations and terminology as in $[17,20]$.

\section{Preliminaries}

In this section, we give some definitions and state some previous results. Let

$$
S^{m-1}=\left\{x \in R^{m}: x_{i} \geq 0, \sum_{i=1}^{m} x_{i}=1\right\}
$$

be an $(m-1)$-dimensional simplex. Then the vectors

$$
e_{k}=(0,0, \ldots, \underbrace{1}_{k}, \ldots, 0), \quad k=\overline{1, m}
$$

are its vertices. For $\alpha \subset I=\{1,2, \ldots, m\}$, the set $F_{\alpha}=\left\{x \in S^{m-1}: x_{i}=0, i \notin \alpha\right\}$ is called a face of the simplex.

For $x=\left(x_{1}, x_{2}, \ldots, x_{m}\right) \in S^{m-1}$, let us put $x_{\downarrow}=\left(x_{[1]}, x_{[2]}, \ldots, x_{[m]}\right)$, where $\left(x_{[1]}, x_{[2]}, \ldots, x_{[m]}\right)-$ nonincreasing rearrangement of $\left(x_{1}, x_{2}, \ldots, x_{m}\right)$, that is, $x_{[1]} \geq x_{[2]} \geq \cdots \geq x_{[m]}$. We say [15] that $x$ is majorized by $y$ on $S^{m-1}$, and write $x \prec y$ (or $y \succ x$ ) if

$$
\sum_{i=1}^{k} x_{[i]} \leq \sum_{i=1}^{k} y_{[i]}, \quad \forall k=\overline{1, m-1}
$$

It is easy to see that for any $x \in S^{m-1}$, we have

$$
\left(\frac{1}{m}, \frac{1}{m}, \ldots, \frac{1}{m}\right) \prec x \prec(1,0,0, \ldots, 0) .
$$


Definition 1 An operator $V: S^{m-1} \rightarrow S^{m-1}$ is called dissipative if

$$
V x \succ x, \quad \forall x \in S^{m-1} .
$$

More information on dissipative operators on $S^{m-1}$ can be found in [17]. Now, let us recall some terminology. Let $x^{0} \in S^{m-1}$ and $V: S^{m-1} \rightarrow S^{m-1}$ be an operator. Then the set $\left\{x^{0}, V x^{0}, V^{2} x^{0}, \ldots\right\}$ is called the trajectory of $V$ starting at the point $x^{0}$. The point $x^{0}$ satisfying $V x^{0}=x^{0}$ is called fixed. The set of all fixed points of the q.s.o. $V$ is denoted by $\operatorname{Fix}(V)$. A q.s.o. $V: S^{m-1} \rightarrow S^{m-1}$ is called regular if the trajectory of any $x \in S^{m-1}$ converges to a unique fixed point. We may note that regular q.s.o. a priori must have a unique fixed point. Let $V$ be a q.s.o. Then the set $\omega\left(x^{0}\right)=\bigcap_{k \geq 0} \overline{\bigcup_{n \geq k}\left\{V^{n} x^{0}\right\}}$ is called an $\omega$-limit set of trajectory of initial point $x^{0} \in S^{m-1}$. From the compactness of the simplex, one can deduce that $\omega\left(x^{0}\right) \neq \emptyset$ for all $x^{0} \in S^{m-1} . V$ is called ergodic if the following limit exists $\lim _{n \rightarrow \infty} \frac{x+V x+\cdots+V^{n-1} x}{n}$ for any $x \in S^{m-1}$.

The following facts are known for dissipative q.s.o. on $S^{m-1}$.

\section{Theorem $2.1[17,20]$}

- Any dissipative operator is ergodic.

- Any dissipative q.s.o. has either unique or infinitely many fixed points.

- One of the following statements always holds for a dissipative q.s.o.

- The operator is regular. Its unique point is either a vertex of the simplex or the center of its face.

- The operator has infinitely many fixed points. $\omega$-Limit set of any initial point is contained in the set of fixed points, i.e., $\omega(x) \subset \operatorname{Fix}(V)$.

In the next section, we define a dissipative operator on infinite-dimensional simplex and study the statements above in infinite-dimensional setting.

\section{Infinite-dimensional dissipative operators}

In this section, we define dissipative quadratic stochastic operators on infinite-dimensional simplex. We study some properties and examples of dissipative q.s.o.

Let $\ell_{1}$ be the set of absolutely summable sequences. The set

$$
S=\left\{x=\left(x_{1}, x_{2}, \ldots\right) \in l_{1}: x_{i} \geq 0, \sum_{i=1}^{\infty} x_{i}=1\right\}
$$

is called an infinite-dimensional simplex. The $\ell_{1}$ norm is defined as $\|x\|_{1}=\sum_{i=1}^{\infty}\left|x_{i}\right|$. So, (1) can be rewritten as

$$
S=\left\{x=\left(x_{1}, x_{2}, \ldots\right) \in l_{1}: x_{i} \geq 0,\|x\|_{1}=1\right\} .
$$

It is known that $S=\overline{\operatorname{co}(\operatorname{Extr}(S))}$. Moreover, any extreme point of $S$ has the following form

$$
e_{k}=(0,0, \ldots, 1,0, \ldots)
$$

where 1 stands in $k$ th position. That is, vertices of the simplex are extreme points of the simplex. An infinite-dimensional quadratic stochastic operator (q.s.o. for shortness) $V$ : 
$S \rightarrow S$ is defined in the following way

$$
(V x)_{k}=\sum_{i, j=1}^{\infty} p_{i j, k} x_{i} x_{j}, \quad k=1,2, \ldots
$$

where the coefficients $p_{i j, k}$, satisfy the following conditions

$$
p_{i j, k}=p_{j i, k} \geq 0, \quad \sum_{k=1}^{\infty} p_{i j, k}=1
$$

One can easily see that the sum (2) is convergent. It is also important to note that the operator (2) is well defined, that is, it maps simplex into itself.

For a point $x=\left(x_{1}, x_{2}, \ldots\right)$, from $S$, let $x_{\downarrow}=\left(x_{[1]}, x_{[2]}, \ldots\right)$ be a nonincreasing rearrangement of $x$, that is, $x_{[1]} \geq x_{[2]} \geq \cdots$. Recall that for two elements $x, y$, taken from the simplex $S$, we say that $x$ is majorized by $y$, and write $x \prec y$ if the following holds

$$
\sum_{i=1}^{k} x_{[i]} \leq \sum_{i=1}^{k} y_{[i]}, \quad \forall k=1,2, \ldots
$$

This definition of majorization is given in [23]. General definition of majorization differs from the one that is given above in [23]; however, on $\ell_{1}$, we can give as above.

Definition 2 An operator $V: S \rightarrow S$ is called dissipative if

$$
V x \succ x, \quad \forall x \in S .
$$

Lemma 3.1 Let $V$ be a linear dissipative operator, that is $V x=A x$, where $A=\left(a_{i j}\right)_{i, j \in N}$ is an infinite matrix. Then $A$ is $(0,1)$ column stochastic matrix.

Note that here and henceforth $N$ denotes natural numbers.

Proof of Lemma 3.1 Since $V x \succ x$, then by putting $x=e_{i}$ we have $A e_{i} \succ e_{i}$. At the same time, it is easy to see that $A e_{i} \prec e_{i}$. That is why $\left(A e_{i}\right)_{\downarrow}=\left(e_{i}\right)_{\downarrow}$, which means that only one component of the vector $A e_{i}$ is 1 , and the others are 0 . Therefore, the matrix $A$ is $(0,1)$ column stochastic matrix.

From this lemma, it follows that the class of linear dissipative operators on $S$ is not large. Therefore, we are interested to study nonlinear (that is q.s.o.) dissipative operators. Let us provide some examples of dissipative quadratic operators.

Example 1 Let $V: S^{m-1} \rightarrow S^{m-1}$ be a finite-dimensional dissipative q.s.o., then the operator $W: S \rightarrow S$, given by

$$
\left.\begin{array}{l}
(W x)_{k}=(V x)_{k}, \quad k=\overline{1, m}, \\
(W x)_{k}=0, \quad k>m,
\end{array}\right\}
$$

is an evidently infinite-dimensional dissipative q.s.o. Because $V$ is dissipative, then the conditions (4) can easily be verified. 
Example 2 Let $V: S^{m-1} \rightarrow S^{m-1}$ be a finite-dimensional dissipative q.s.o., then the operator $W: S \rightarrow S$, given by

$$
\left.\begin{array}{l}
(W x)_{k}=(V x)_{k}+x_{k} \sum_{i=m+1}^{\infty} x_{i}, \quad k=\overline{1, m}, \\
(W x)_{k+m}=x_{k+m}, \quad k \in N
\end{array}\right\}
$$

is a dissipative q.s.o. Dissipativity can be verified by using the fact that $V$ is dissipative.

We can also provide some more examples of a dissipative q.s.o. by pointing out that if an operator is dissipative, then by rearranging its components, it preserves its dissipativity. Let us consider the operator $V: S \rightarrow S$, given as follows

$$
V\left(x_{1}, x_{2}, \ldots\right)=\left(0, x_{1}, x_{2}, \ldots\right)
$$

One can see that this operator maps infinite-dimensional simplex into itself, and since $(V x)_{\downarrow}=x_{\downarrow}$, then $V$ is dissipative. One can see that $V$ does not have nonzero fixed points. As we consider $V$ acting on $S$, then $V$ has no fixed points. Therefore, Theorem 2.1 fails in infinite-dimensional setting.

Now, let us put $x=(1,0,0, \ldots)$ and calculate the following Ceasaro mean

$$
\frac{x+V x+\cdots+V^{n-1} x}{n}=\left(\frac{1}{n}, \frac{1}{n}, \ldots, \frac{1}{n}, 0, \ldots\right) .
$$

Let us consider $l_{1}$ norm and denote it by $\|\cdot\|_{1}$. Note that if a sequence on $l_{1}$ converges in its norm, then it converges componentwise. So, if the sequence $\left(\frac{1}{n}, \frac{1}{n}, \ldots, \frac{1}{n}, 0, \ldots\right)$ converges, it converges to $(0,0, \ldots)$. But it can easily be seen that

$$
\left\|\left(\frac{1}{n}, \frac{1}{n}, \ldots, \frac{1}{n}, 0, \ldots\right)-(0,0, \ldots)\right\|_{1}=1 .
$$

Therefore, the average

$$
\lim _{n \rightarrow \infty} \frac{x+T x+\cdots+T^{n-1} x}{n}
$$

does not have a limit. Thus, for dissipative q.s.o., an ergodic theorem fails dramatically. In addition to that, one can see that the trajectory of certain point under $V$ may not converge in general. Indeed, take $x=e_{1}=(1,0,0, \ldots)$, then $V^{n} x=e_{n}\left(e_{n}\right.$ is $n$th vertex of the simplex $S$ ), and hence $\left\|V^{n} x-V^{n+m} x\right\|_{1}=\left\|e_{n}-e_{n+m}\right\|_{1}=2$. So, the trajectory is divergent. We see that when we consider operators in infinite-dimensional space, all the statements in Theorem 2.1 fail dramatically. This is the difference between finite-dimensional and infinite-dimensional cases.

We now study some properties of dissipative q.s.o.

Given q.s.o. $V$, we denote $a_{i j}=\left(p_{i j, 1}, p_{i j, 2}, \ldots, p_{i j, m}, \ldots\right) \forall i, j \in N$, where $p_{i j, k}$ are the coefficients of q.s.o. $V$. One can see that $a_{i j} \in S$, for all $i, j \in N$.

Lemma 3.2 Let $V$ be a dissipative q.s.o. Then the following conditions hold

$$
\left(a_{i i}\right)_{\downarrow}=e_{1} \quad \forall i \in N .
$$


Proof Due to dissipativity of $V$ one has $V x \succ x, \forall x \in S$. Now by putting $x=e_{i}$ we get $e_{i} \prec V e_{i}$. On the other hand, we have $e_{i} \succ x, \forall x \in S$. That is why $\left(V e_{i}\right)_{\downarrow}=\left(e_{i}\right)_{\downarrow}=e_{1}$. Then the equality $V e_{i}=a_{i i}$ implies the assertion.

The lemma above implies that any dissipative q.s.o. can be written as

$$
(V x)_{k}=\sum_{i \in \alpha_{k}} x_{i}^{2}+2 \sum_{i<j} p_{i j, k} x_{i} x_{j}, \quad k \in N,
$$

where

$$
\alpha_{k} \subset N, \quad \alpha_{i} \cap \alpha_{j}=\emptyset, \quad i \neq j, \quad \bigcup_{k=1}^{\infty} \alpha_{k}=N
$$

We call (5) a canonical form of dissipative q.s.o. $V$.

Lemma 3.3 Let (5) be a dissipative q.s.o.

(i) If $j \in \alpha_{k_{0}}$, then $p_{i j, k_{0}}=\left(a_{i j}\right)_{[1]} \geq \frac{1}{2}, \forall i \in N$.

(ii) For any $k \geq 3$, one has $\left(a_{i j}\right)_{[k]}=0, \forall i \in N$.

Proof (i) Let $j \in \alpha_{k_{0}}$ and $x=(1-\lambda) e_{j}+\lambda e_{i}$, where $e_{i}, e_{j}$ are the vertices of the simplex and $0 \leq \lambda \leq 1$. We can choose $\lambda$ sufficiently small so that $x_{[1]}=1-\lambda$ and $(V x)_{[1]}=(V x)_{k_{0}}$. Since $V x>x$, then $x_{[1]} \leq(V x)_{[1]}$, so $1-\lambda \leq(V x)_{k_{0}}$ or

$$
1-\lambda \leq(1-\lambda)^{2}+2 p_{i j, k_{0}} \lambda(1-\lambda)
$$

The last inequality implies that $p_{i j, k_{0}} \geq \frac{1}{2}$. Since $i$ is chosen arbitrary, then the above is true for all $i \in N$.

(ii) Denote $p_{i j, k^{*}}=\max _{t \neq k_{0}} p_{i j, t}$. This maximum exists as the coefficients of q.s.o. are not greater than 1 . One can see that $(V x)_{k^{*}}=\left(a_{i j}\right)_{[2]}$. Now, from

$$
x_{[1]}+x_{[2]} \leq(V x)_{[1]}+(V x)_{[2]},
$$

we obtain

$$
1 \leq(1-\lambda)^{2}+2\left(p_{i j, k_{o}}+p_{i j, k^{*}}\right) \lambda(1-\lambda)
$$

From this inequality, we get $p_{i j, k_{o}}+p_{i j, k^{*}} \geq \frac{2 \lambda-\lambda^{2}}{2 \lambda(1-\lambda)}=\frac{2-\lambda}{2(1-\lambda)}=\frac{1}{2}\left(1+\frac{1}{1-\lambda}\right) \geq 1$. This yields $p_{i j, k_{o}}+$ $p_{i j, k^{*}}=1$ and $\left(a_{i j}\right)_{[k]}=0 \forall k \geq 3, \forall i \in N$.

\section{The limit behavior of the trajectories}

In this section, we study the limit behavior of the trajectories of dissipative q.s.o. We study some criteria for the existence of fixed point. We also provide some examples of regular dissipative q.s.o.

Theorem 4.1 A dissipative q.s.o. $V$ defined on infinite-dimensional simplex $S$ has either 0 or 1 or infinitely many fixed points.

The proof is based on expressing $V$ in canonical form, dividing the proof into several cases and using proof method of its finite-dimensional counterpart. 
Proof of Theorem 4.1 First, we rewrite dissipative q.s.o. in its canonical form and correspond the partition $\left\{\alpha_{k}, k \in N\right\}$ of $N$ to a dissipative q.s.o. $V$.

We need to know those numbers $k$ for which $k \in \alpha_{k}$. Hence we consider the following possible cases

(1) There is no $k$ such that $k \in \alpha_{k}$, that is, $k \notin \alpha_{k}, \forall k \in N$.

(2) There exists numbers $\left\{k_{i}, i \in N\right\}$ such that $k_{i} \in \alpha_{k_{i}}, \forall i \in N$.

(1) Since there is no $k$ with $k \in \alpha_{k}$ and $\alpha_{k}$ is a partition of $N$, then a particular number $k_{1}$ must belong to one of the set other than $\alpha_{k_{1}}$, say $\alpha_{k_{2}}$. The number $k_{2}$ belongs to some $\alpha_{k_{3}}$ and so on. Therefore, there exists a sequence $K=\left\{k_{l}, l \in N\right\}$ such that $k_{l} \in \alpha_{\pi\left(k_{l}\right)}$, where $\pi$ is a bijection on the set $K=\left\{k_{l}, l \in N\right\}$. In this case, the operators $V$ can be written as follows

$$
\left.\begin{array}{l}
(V x)_{k_{l}}=x_{\pi\left(k_{l}\right)}^{2}+\sum_{i \in \alpha_{k} \backslash\left\{\pi\left(k_{l}\right)\right\}} x_{i}^{2}+\sum_{i<j} 2 p_{i j, k_{l}} x_{i} x_{j}, \quad k_{l} \in K, \\
(V x)_{k}=\sum_{i \in \alpha_{k}} x_{i}^{2}+2 \sum_{i<j} p_{i j, k} x_{i} x_{j}, \quad k \in N \backslash K .
\end{array}\right\}
$$

Taking into account $\sum_{i=1}^{\infty} x_{i}=1$, one can rewrite (7) as

$$
\left.\begin{array}{l}
(V x)_{k_{l}}=x_{\pi\left(k_{l}\right)}+\sum_{i \in \alpha_{k_{l}} \backslash\left\{\pi\left(k_{l}\right)\right\}} x_{i}^{2}+\sum_{i<j} 2 p_{i j, k_{l}} x_{i} x_{j}-\sum_{i=1, i \neq \pi\left(k_{l}\right)}^{\infty} x_{i} x_{\pi\left(k_{l}\right)}, \\
\quad k_{l} \in K, \\
(V x)_{k}=\sum_{i \in \alpha_{k}} x_{i}^{2}+2 \sum_{i<j} p_{i j, k} x_{i} x_{j}, \quad k \in N \backslash K .
\end{array}\right\}
$$

The set $K$ can be finite or infinite. We assume that the set $K$ is the largest set, for which $k_{l} \in \alpha_{\pi\left(k_{l}\right)}, \forall k_{l} \in K$. In this case, one can show that $\alpha_{k}=\emptyset, \forall k \in N \backslash K$. Indeed, clearly numbers elements of $K$ do not belong to any of the sets $\alpha_{k_{l}}, k_{l} \in N$. This implies that it is possible to find finite or infinite sequence $K^{\prime}=\left\{k_{l}^{\prime}, l \in N\right\}$ such that $k_{l}^{\prime} \in \alpha_{\pi^{\prime}\left(k_{k}^{\prime}\right)}$, where $\pi^{\prime}$ is a bijection on $K^{\prime}$. But this implies that we found the set $\left(K \cup K^{\prime}\right)$ larger than $K$ with the property that $k_{l} \in \alpha_{\pi\left(k_{l}\right)} \forall k_{l} \in K \cup K^{\prime}$, which is the contradiction. Therefore, one can consider $\alpha_{k}=\emptyset, \forall k \in N \backslash K$.

Now, if we define

$$
L_{k_{l}}=\sum_{i<j} 2 p_{i j, k_{l}} x_{i} x_{j}-\sum_{i=1, i \neq \pi\left(k_{l}\right)}^{\infty} x_{i} x_{\pi\left(k_{l}\right)}, \quad k_{l} \in K
$$

then one can show that $L_{k_{l}} \geq 0, \forall k_{l} \in K$. Indeed, since $\pi\left(k_{l}\right) \in \alpha_{k_{l}}, k_{l} \in K$, then Lemma 3.3 implies that $2 p_{i \pi\left(k_{l}\right), k_{l}} \geq 1$ for all $i \in N \backslash \pi\left(k_{l}\right)$. Therefore,

$$
\begin{aligned}
L_{k_{l}}= & 2 p_{1 \pi\left(k_{l}\right), k_{l}} x_{1} x_{k_{l}}+2 p_{2 \pi\left(k_{l}\right), k_{l}} x_{1} x_{k_{l}}+\cdots \\
& +\sum_{i<j, j \neq \pi\left(k_{l}\right)} 2 p_{i j, k_{l}} x_{i} x_{j}-\left(x_{1} x_{\pi\left(k_{l}\right)}+x_{2} x_{\pi\left(k_{l}\right)}+x_{\pi\left(k_{l}\right)-1} x_{\pi\left(k_{l}\right)}+x_{\pi\left(k_{l}\right)+1} x_{\pi\left(k_{l}\right)}+\cdots\right) \\
= & \left(2 p_{1 \pi\left(k_{l}\right), k_{l}}-1\right) x_{1} x_{k_{l}}+\left(2 p_{2 \pi\left(k_{l}\right), k_{l}}-1\right) x_{1} x_{k_{l}}+\cdots+\sum_{i<j, j \neq \pi\left(k_{l}\right)} 2 p_{i j, k_{l}} x_{i} x_{j} \geq 0 .
\end{aligned}
$$

Let $V x=x$. Using $L_{k_{l}} \geq 0$, and summing up (8) for all values of $k_{l}$, one gets

$$
\sum_{k_{l} \in K} \sum_{i \in \alpha_{k_{l}} \backslash\left\{\pi\left(k_{l}\right)\right\}} x_{i}^{2}=0
$$


Note that since $\left\{\alpha_{k}, k \in N\right\}$ is the partition of $N$, then

$$
\sum_{k \in N} \sum_{i \in \alpha_{k}} x_{i}^{2}=\sum_{i \in N} x_{i}^{2}
$$

and since $\alpha_{k}=\emptyset, \forall k \in N \backslash K$, one finds

$$
\sum_{k \in K} \sum_{i \in \alpha_{k}} x_{i}^{2}=\sum_{i \in N} x_{i}^{2}
$$

Therefore,

$$
0=\sum_{k_{l} \in K} \sum_{i \in \alpha_{k_{l}} \backslash\left\{\pi\left(k_{l}\right)\right.} x_{i}^{2}=\sum_{k \in K} \sum_{i \in \alpha_{k}} x_{i}^{2}-\sum_{k \in K} x_{i}^{2}=\sum_{k \in N} x_{i}^{2}-\sum_{k \in K} x_{i}^{2}=\sum_{k \in N \backslash K} x_{i}^{2},
$$

which implies that $x_{k}=0, \forall k \in N \backslash K$. In addition, from (8), it follows that all $x_{k_{l}}, k_{l} \in K$ should be equal. So, if $K$ is a finite set, then $V$ has a unique fixed point, which is

$$
x= \begin{cases}x_{i}=\frac{1}{|K|}, & \text { if } i \in K, \\ x_{i}=0, & \text { otherwise. }\end{cases}
$$

If $K$ is an infinite set, then all components of a fixed point should be 0 , therefore, there are no fixed points.

(2) Let $F$ be the set of numbers $k_{l}$ such that $k_{l} \in \alpha_{k_{l}}$ for all $k_{l} \in F$.

Since there is no $k \in N \backslash F$ with $k \in \alpha_{k}$ and $\alpha_{k}$ is a partition of $N$, then a particular number $k_{1}$ must belong to one of the set other than $\alpha_{k_{1}}$, say $\alpha_{k_{2}}$. The number $k_{2}$ belongs to some $\alpha_{k_{3}}$ and so on. Therefore, there exists a sequence $K=\left\{k_{i}, i \in N\right\}$ such that $k_{i} \in \alpha_{\pi\left(k_{i}\right)}$, where $\pi$ is a bijection on the set $K=\left\{k_{i}, i \in N\right\}$. In this case, the operators $V$ can be written as follows

$$
\left.\begin{array}{l}
(V x)_{k_{l}}=x_{k_{l}}^{2}+\sum_{i \in \alpha_{k_{l}} \backslash k_{l}} x_{i}^{2}+\sum_{i<j} 2 p_{i j, k_{l}} x_{i} x_{j}, \quad k_{l} \in F, \\
(V x)_{k_{l}}=x_{\pi\left(k_{l}\right)}^{2}+\sum_{i \in \alpha_{k_{l}} \backslash\left\{\pi\left(k_{l}\right)\right\}} x_{i}^{2}+\sum_{i<j} 2 p_{i j, k_{l}} x_{i} x_{j}, \quad k_{l} \in K, \\
(V x)_{k}=\sum_{i \in \alpha_{k}} x_{i}^{2}+2 \sum_{i<j} p_{i j, k} x_{i} x_{j}, \quad k \in N \backslash F \cup K .
\end{array}\right\}
$$

Taking into account $\sum_{i=1}^{\infty} x_{i}=1$, one can rewrite (10) as:

$$
\left.\begin{array}{rl}
(V x)_{k_{l}=} & x_{k_{l}}+\sum_{i \in \alpha_{k_{k}} \backslash\left\{k_{l}\right\}} x_{i}^{2}+\sum_{i<j} 2 p_{i j, k_{l}} x_{i} x_{j} \\
& -\sum_{i=1, i \neq k_{l}}^{\infty} x_{i} x_{k_{l}}, \quad k_{l} \in F, \\
(V x)_{k_{l}}= & x_{\pi\left(k_{l}\right)}+\sum_{i \in \alpha_{k} \backslash\left\{\pi\left(k_{l}\right)\right\}} x_{i}^{2}+\sum_{i<j} 2 p_{i j, k_{l}} x_{i} x_{j} \\
& -\sum_{i=1, i \neq \pi\left(k_{l}\right)}^{\infty} x_{i} x_{\pi\left(k_{l}\right)}, \quad k_{l} \in K, \\
(V x)_{k}= & \sum_{i \in \alpha_{k}} x_{i}^{2}+2 \sum_{i<j} p_{i j, k} x_{i} x_{j}, \quad k \in N \backslash F \cup K .
\end{array}\right\}
$$

Both sets $F$ and $K$ can be finite or infinite, and note that $F \neq \emptyset$, otherwise, the case would coincide with the previous case. We also assume that the sets $F$ and $K$ are largest sets satisfying conditions given in their definitions. Therefore, similar to a previous case one can show that $\alpha_{k}=\emptyset, k \in N \backslash F \cup K$.

Now, if we define $L_{k_{l}}, k_{l} \in F \cup K$ as in (9), one can show in the same way that $L_{k_{l}} \geq 0$, $\forall k_{l} \in F \cup K$. Therefore, by letting $V x=x$ and summing up (10) for all values of $k_{l} \in F \cup K$, 
one gets

$$
\sum_{k_{l} \in F} \sum_{i \in \alpha_{k_{l}} \backslash\left\{k_{l}\right\}} x_{i}^{2}+\sum_{k_{l} \in K} \sum_{i \in \alpha_{k_{l}} \backslash\left\{\pi\left(k_{l}\right)\right\}} x_{i}^{2}=0 .
$$

Note that $\left\{\alpha_{k}, k \in N\right\}$ is the partition of $N$, hence

$$
\sum_{k \in F \cup N} \sum_{i \in \alpha_{k}} x_{i}^{2}=\sum_{i \in N} x_{i}^{2}
$$

taking into account $\alpha_{k}=\emptyset, \forall k \in N \backslash(F \cup K)$, we find

$$
\sum_{k \in F \cup K} \sum_{i \in \alpha_{k}} x_{i}^{2}=\sum_{i \in N} x_{i}^{2}
$$

Therefore,

$$
\begin{aligned}
0 & =\sum_{k_{l} \in F} \sum_{i \in \alpha_{k_{l}} \backslash\left\{k_{l}\right\}} x_{i}^{2}+\sum_{k_{l} \in K} \sum_{i \in \alpha_{k_{l}} \backslash\left\{\pi\left(k_{l}\right)\right\}} x_{i}^{2} \\
& =\sum_{k \in F \cup K} \sum_{i \in \alpha_{k}} x_{i}^{2}-\sum_{k \in F \cup K} x_{i}^{2}=\sum_{k \in N} x_{i}^{2}-\sum_{k \in F \cup K} x_{i}^{2}=\sum_{k \in N \backslash(F \cup K)} x_{i}^{2},
\end{aligned}
$$

which implies that $x_{k}=0, \forall k \in N \backslash K$. In addition, it follows that all $x_{k_{l}}, k_{l} \in K$ are equal.

Due to the operator above, (11) can be simplified as follows

$$
\left.\begin{array}{l}
(V x)_{k_{l}}=x_{k_{l}}+L_{k_{l}}, \quad k_{l} \in F, \\
(V x)_{k_{l}}=x_{\pi\left(k_{l}\right)}+L_{k_{l}}, \quad k_{l} \in K, \\
(V x)_{k}=\sum_{i, j=1}^{\infty} p_{i j, k} x_{i} x_{j}, \quad k \in N \backslash(F \cup N) .
\end{array}\right\}
$$

Using (12), one can find all the fixed points by putting $V x=x$ and solving the system of equations. We consider the following few cases. First of all, note that $|F|>0$ and $|K|>1$ (here $|F|$ stands for the cardinality of $F$ ).

If $|F|=1$ (say, $F=\{1\}$ ) and $K$ is finite (say, $K=\{2,3, \ldots, k+1\}$ ), then

$$
\operatorname{Fix}(V)=\{(\alpha, \beta, \beta, \ldots, \beta, 0,0, \ldots) \mid \alpha+k \beta=1,0 \leq \alpha, \beta \leq 1\},
$$

where $\beta$ appears $k$ times.

If $|F|=1$ (say, $F=\{1\}$ ) and $K$ is infinite, then since all $x_{k_{l}}, k_{l} \in K$ are equal and $\sum_{k_{l} \in K} x_{k_{l}} \leq$ 1 implies that $x_{k_{l}}=0$ for all $k_{l} \in K$. Therefore, the operator has a unique fixed point $(1,0,0, \ldots)$.

If $F$ is finite (say, $F=\{1,2, \ldots, k\}$ ) and $K$ is finite (say, $K=k+1, k+2, \ldots, k+m$ ), then

$$
\operatorname{Fix}(V)=\{(\alpha, \alpha, \ldots, \alpha, \beta, \beta, \ldots, \beta, 0,0, \ldots) \mid k \alpha+m \beta=1,0 \leq \alpha, \beta \leq 1\}
$$

If $F$ is finite (say, $F=\{1,2, \ldots, k\}$ ) and $K$ is infinite, then the unique fixed point is $\left(\frac{1}{k}, \frac{1}{k}, \ldots, \frac{1}{k}, 0,0, \ldots\right)$.

Finally, if both $F$ and $K$ are infinite, then the vertices $e_{k_{l}}, k_{l} \in F$ of the simplex are fixed. So, in this case, there are infinitely many fixed points since $F$ is infinite. 
Corollary 4.2 Let dissipative quadratic operator $V$ be given in canonical form (5). Then $V$ has a fixed point if and only if there exists a finite sequence $k_{1}, k_{2}, \ldots, k_{l}(l \geq 1)$ such that $k_{l} \in \alpha_{\pi\left(k_{l}\right)}$ for some permutation $\pi$ of the sequence $\left\{k_{l}, l \geq 1\right\}$.

Proof follows from the prof of Theorem 4.1.

Now, we study the limit behavior of the trajectories. We have seen in Section 3 that the trajectory of the point under dissipative operator may not converge in general. Here, we consider a particular case, assuming that a dissipative operator has a unique fixed point.

Theorem 4.3 If dissipative q.s.o. $V: S \rightarrow S$ has a unique fixed point, then the operator is regular at this point, i.e., the trajectory of any initial point tends to this unique point.

Proof Let us use the decomposition of $V$ given by (11), and let $F$ and $K$ be the sets defined in (11). We have seen in the previous Theorem 4.1 that an operator has a unique fixed point if and only if one of the conditions is satisfied: (1) $|F|=1, K=\emptyset$, (2) $F=\emptyset,|K|<\infty$.

Let us consider the case (1) and assume that $F=\{1\}$. Then $V$ has a form

$$
\left.\begin{array}{l}
(V x)_{1}=x_{1}^{2}+\sum_{i \in \alpha_{1} \backslash\{1\}} x_{i}^{2}+\sum_{i, j=1}^{\infty} 2 p_{i j, 1} x_{i} x_{j}, \\
(V x)_{k}=\sum_{i \in \alpha_{i}} x_{i}^{2}+\sum_{i, j=1}^{\infty} 2 p_{i j, k} x_{i} x_{j}, \quad k \neq 1 .
\end{array}\right\}
$$

Since the sets $F$ and $K$ are chosen as largest, then we can simplify the operator as

$$
\left.\begin{array}{l}
(V x)_{1}=x_{1}+\sum_{i \in \alpha_{1} \backslash\{1\}} x_{i}^{2}+\sum_{i, j=1}^{\infty} 2 p_{i j, 1} x_{i} x_{j}-\sum_{i=2}^{\infty} x_{1} x_{i}, \\
(V x)_{k}=\sum_{i, j=1}^{\infty} 2 p_{i j, k} x_{i} x_{j}, \quad k \neq 1 .
\end{array}\right\}
$$

Note that we set $\alpha_{k}=\emptyset, k \geq 2$.

Let us set $x^{(n)}=V^{(n)} x$ and define $\varphi(x)=x_{1}$. Because Lemma 3.3 implies that $\sum_{i, j=1}^{\infty} 2 \times$ $p_{i j, 1} x_{i} x_{j}-\sum_{i=2}^{\infty} x_{1} x_{i} \geq 0$, one can see that $\varphi\left(x^{(1)}\right) \geq \varphi(x)$, which means that $\varphi\left(x^{(n)}\right), n \in N$ is monotone and bounded sequence, and hence convergent. We put $\lim _{n \rightarrow \infty} \varphi\left(x^{(n)}\right)=C$.

Note that from (13), by applying iterations $n$ times to its first equation, we get

$$
x_{1}^{(n+1)}=x_{1}^{(n)}+\sum_{i \in \alpha_{1} \backslash\{1\}}\left(x_{i}^{(n)}\right)^{2}+\sum_{i, j=1}^{\infty} 2 p_{i j, 1} x_{i}^{(n)} x_{j}^{(n)}-\sum_{i=2}^{\infty} x_{1}^{(n)} x_{i}^{(n)} .
$$

Now, since $\lim _{n \rightarrow \infty} x_{1}^{(n+1)}=\lim _{n \rightarrow \infty} x_{1}^{(n)}=C$, then

$$
\sum_{i \in \alpha_{1} \backslash\{1\}}\left(x_{i}^{(n)}\right)^{2}=\sum_{i=2}^{\infty}\left(x_{i}^{(n)}\right)^{2}=0,
$$

hence $x_{i}^{(n)} \rightarrow 0$ for all $i \geq 2$ as $n \rightarrow \infty$. From the last, one can find that $x_{1}^{(n)} \rightarrow 1$ as $n \rightarrow \infty$. Finally, from

$$
\left\|x^{(n)}-e_{1}\right\|_{1}=1-x_{1}^{(n)}+x_{2}^{(n)}+x_{3}^{(n)}+\cdots=2\left(1-x_{1}^{(n)}\right) \rightarrow 0
$$

as $n \rightarrow \infty$, we find that the trajectory of any initial point converges to $e_{1}$. 
Now, we turn to the other case. Let $F=\emptyset$ and $K=\{1,2, \ldots, l\}$. The unique point in this case is $\left(\frac{1}{l}, \frac{1}{l}, \ldots, \frac{1}{l}, 0,0, \ldots\right)$. The operator has a form (7) or (8). Note that one can assume $\pi(i)=i+1, \pi(k)=1$. Therefore, the operator has a form

$$
\left.\begin{array}{l}
(V x)_{k}=x_{k+1}+\sum_{i \in \alpha_{k} \backslash\{k+1\}} x_{i}^{2}+\sum_{i<j} 2 p_{i j, k} x_{i} x_{j}-\sum_{i \in N, i \neq k+1} x_{i} x_{k+1}, \\
1 \leq k \leq l-1, \\
(V x)_{l}=x_{1}+\sum_{i \in \alpha_{n} \backslash\{1\}} x_{i}^{2}+\sum_{i<j} 2 p_{i j, l} x_{i} x_{j}-\sum_{i=2}^{\infty} x_{i} x_{1}, \\
(V x)_{k}=\sum_{i \in \alpha_{k}} x_{i}^{2}+2 \sum_{i<j} p_{i j, k} x_{i} x_{j}, \quad k \geq l+1 .
\end{array}\right\}
$$

Define

$$
\varphi(x)=x_{1}+x_{2}+\cdots+x_{l}
$$

Then

$$
\varphi(V x)=\sum_{i=1}^{l}(V x)_{i}=\sum_{i=1}^{l} x_{i}+\sum_{k=1}^{l} \sum_{i \in \alpha_{k} \backslash\{k+1\}} x_{i}^{2}+\sum_{i=1}^{l} L_{i} .
$$

Here, $L_{i}$ satisfy (9). Since $L_{i} \geq 0$, then from the above, it follows that $\varphi(V x) \geq \varphi(x)$. Hence, the sequence $\left\{\varphi\left(x^{(n)}\right): n=1,2, \ldots\right\}$ is non-decreasing and bounded. That is why the limit $\lim _{n \rightarrow \infty} \varphi\left(x^{(n)}\right)$ exists. Let us put $C=\lim _{k \rightarrow \infty} \varphi\left(x^{(n)}\right)$.

Since $K$ is chosen as largest, then one can rewrite the above as

$$
\varphi(V x)=\sum_{i=1}^{l}(V x)_{i}=\sum_{i=1}^{l} x_{i}+\sum_{i=l+1}^{\infty} x_{i}^{2}+\sum_{i=1}^{l} L_{i} .
$$

From the last, we get

$$
\begin{aligned}
\varphi\left(x^{(n+1)}\right) & =\sum_{i=1}^{l}\left(x^{(n+1)}\right)_{i}=\sum_{i=1}^{l}\left(x^{(n)}\right)_{i}+\sum_{i=l+1}^{\infty}\left(x^{(n)}\right)_{i}^{2}+\sum_{i=1}^{l} L_{i}^{(n)} \\
& =\varphi\left(x^{(n)}\right)+\sum_{i=l+1}^{m}\left(x^{(n)}\right)_{i}^{2}+\sum_{i=1}^{l} L_{i}^{(n)},
\end{aligned}
$$

where

$$
L_{s}^{(n)}=\sum_{i<j} 2 p_{i j, s}\left(x^{(n)}\right)_{i}\left(x^{(n)}\right)_{j}-\sum_{i \in N, i \neq s+1}\left(x^{(n)}\right)_{i}\left(x^{(n)}\right)_{s+1}, \quad s=\overline{1, l}, n=1,2, \ldots
$$

Since $\lim _{n \rightarrow \infty} \varphi\left(x^{(n)}\right)=\lim _{n \rightarrow \infty} \varphi\left(x^{(n)}\right)=C$ and $L_{s}^{(n)} \geq 0$, then we get $\sum_{i=l+1}^{m}\left(x^{(n)}\right)_{i}^{2} \rightarrow 0$ as $n \rightarrow \infty$. Therefore, $\left(x^{(n)}\right)_{i} \rightarrow 0$ for all $i=\overline{l+1, \infty}$, as $n \rightarrow \infty$. Taking into account $\sum_{i=1}^{m}\left(V^{k} x\right)_{i}=1$, we get $C=1$. Furthermore, if $n \rightarrow \infty$, then $\sum_{i \in \alpha_{S} \backslash\{s+1\}}\left(x^{(n)}\right)_{i}^{2} \rightarrow 0$ for $s=\overline{1, l}$ and $L_{i}^{k} \rightarrow 0$. Therefore, from

$$
\left(x^{(n+1)}\right)_{i}=\left(x^{(n)}\right)_{i+1}+\sum_{i \in \alpha_{i} \backslash\langle i+1\}}\left(x^{(n)}\right)_{i}^{2}+L_{i}^{n}, \quad 1 \leq i \leq l,
$$


we get

$$
\lim _{n \rightarrow \infty}\left(x^{(n)}\right)_{i+1}=\lim _{n \rightarrow \infty}\left(x^{(n)}\right)_{i}
$$

which implies that $\lim _{n \rightarrow \infty}\left(x^{(n)}\right)_{i}=\frac{1}{l}, \forall i=\overline{1, l}$.

Since $x_{i}^{(n)} \rightarrow 0$ as $n \rightarrow 0$ for all $i>l$, then one can assume that the initial point is taken in a small neighborhood of the set $\left\{x \in S \mid \sum_{1}^{l} x_{i}=1\right\}$, and hence

$$
\begin{aligned}
\left\|x^{(n)}-\left(\frac{1}{l}, \frac{1}{l}, \ldots, \frac{1}{l}, 0,0, \ldots\right)\right\|_{1} & =\frac{1}{l}-x_{1}^{(n)}+\frac{1}{l}-x_{2}^{(n)}+\cdots+\frac{1}{l}-x_{l}^{(n)}++x_{l+1}^{(n)}+\cdots \\
& =2\left(1-\sum_{i=1}^{l} x_{i}^{(n)}\right) \rightarrow 0
\end{aligned}
$$

as $n \rightarrow \infty$

\section{Conclusion}

In this paper, we study dissipative q.s.o. defined on infinite-dimensional simplex. In this case, we have some obstacles. First, an infinite-dimensional simplex is not compact in $\ell_{1}$ topology, nor it is compact in a weak topology, which makes the study of limit behavior harder. Moreover, the operator may not have fixed points at all. Despite this, we were able to define the dissipative q.s.o. in such space, and we classified the fixed points of dissipative operators. We also studied the limit behavior of the trajectories in some particular cases that can be an impetus to further studies of dissipative q.s.o. on infinite-dimensional space. There are some questions left unanswered. First, note that simple examples can show that a dissipative q.s.o. is not non-expansive operator, so we can not use the general theorems guaranteeing the convergence of Cesaro means. So the questions is: find necessary and sufficient conditions for a dissipative q.s.o. to be mean ergodic (i.e., Cesaro mean of any initial point converges in $\ell_{1}$ norm). Second, investigate the limit behavior of the trajectories for arbitrary dissipative q.s.o.

\section{Competing interests}

The author declares that he has no competing interests.

Received: 30 April 2013 Accepted: 13 August 2013 Published: 10 September 2013

\section{References}

1. Bernstein, SN: The solution of a mathematical problem concerning the theory of heredity. Ucheniye-Zapiski N.-I. Kaf. Ukr. Otd. Mat. 1, 83-115 (1924) (Russian)

2. Lyubich, Yl: Iterations of quadratic maps. Math. Economics and Functional Analysis. Nauka, Moscow (1974) (Russian)

3. Lyubich, Yl: Mathematical Structures in Population Genetics. Springer, Berlin (1992)

4. Kesten, H: Quadratic transformations: a model for population growth. I. Adv. Appl. Probab. 2, 1-82 (1970)

5. Kesten, H: Quadratic transformations: a model for population growth. II. Adv. Appl. Probab. 2, 179-228 (1970)

6. Vallander, SS: On the limit behavior of iteration sequence of certain quadratic transformations. Sov. Math. Dokl. 13, 123-126 (1972)

7. Zakharevich, MI: On a limit behavior and ergodic hypothesis for quadratic mappings of a simplex. Russ. Math. Surv. 33, 207-208 (1978)

8. Ganikhodzhaev, RN: Quadratic stochastic operators, Lyapunov functions and tournaments. Russian Acad. Sci. Sbornik. Math. 76, 489-506 (1993)

9. Ganikhodzhaev, RN, Eshmamatova, DB: Quadratic authomorphisms of a simplex and asymptotic behaviour of their trajectories. Vladikavkaz Mat. Zh. 2(8), 12-28 (2006) (Russian)

10. Rozikov, UA, Jamilov, UU: On F-quadratic stochastic operators. Math. Notes 4(83), 606-612 (2008)

11. Rozikov, UA, Jamilov, UU: The dynamics of strictly non-Volterra quadratic stochastic operators on $2 \mathrm{D}$ simplex. Mat. Sb. 9(200), 81-94 (2009)

12. Rozikov, UA, Nazir, S: Separable quadratic stochastic operators. Lobachevskii J. Math. 3(31), 215-221 (2010) 
13. Rozikov, UA, Zada, A: On dynamics of $\ell$-Volterra quadratic stochastic operators. Int. J. Biomath. 2(3), 143-159 (2010)

14. Ganikhodzhaev, RN, Mukhamedov, FM, Rozikov, UA: Quadratic stochastic operators: results and open problems. Infin. Dimens. Anal. Quantum Probab. Relat. Top. 2(14), 279-335 (2011)

15. Marshall, A, Olkin, I: Inequalities: Theory of Majorization and Its Applications. Academic Press, New York (1979)

16. Ganikhodzhaev, RN: On the definition of quadratic bistochastic operators. Russ. Math. Surv. 48, $244-246$ (1992)

17. Shahidi, FA: On dissipative quadratic stochastic operators. Appl. Math. Inform. Sci. 2(2), 211-223 (2008)

18. Ganikhodzhaev, RN, Shahidi, FA: Doubly stochastic quadratic operators and Birkhoff's problem. Linear Algebra Appl. 1(432), 24-35 (2010)

19. Shahidi, FA: Doubly stochastic quadratic operators on finite dimensional simplex. Sib. Math. J. 2(50), 463-468 (2009)

20. Shahidi, FA, Abu Osman, MT: The limit behavior of the trajectories of dissipative quadratic stochastic operators on finite dimensional simplex. J. Differ. Equ. Appl. 3(19), 357-371 (2013)

21. Mukhamedov, F, Akin, H, Temir, S: On infinite dimensional quadratic Volterra operators. J. Math. Anal. Appl. 310 533-556 (2005)

22. Shahidi, FA: Necessary and sufficient conditions for doubly stochasticity of infinite-dimensional quadratic operators. Linear Algebra Appl. 438, 96-110 (2013)

23. Markus, AS: Eigenvalues and singular values of the sum and product of linear operators. Russ. Math. Surv. 19, 91-120 (1964)

doi:10.1186/1687-1847-2013-272

Cite this article as: Shahidi: On infinite-dimensional dissipative quadratic stochastic operators. Advances in Difference Equations 2013 2013:272.

\section{Submit your manuscript to a SpringerOpen ${ }^{\circ}$ journal and benefit from:}

- Convenient online submission

- Rigorous peer review

- Immediate publication on acceptance

Open access: articles freely available online

- High visibility within the field

- Retaining the copyright to your article 